\title{
The asymptotic behavior of a dynamical process coming from the development in continuous fractions
}

\author{
Maria-Liliana Bucur ${ }^{1, *}$ and Cristina-Gabriela Cerbulescu $^{2, * *}$ \\ ${ }^{1}$ University of Craiova, Department of Applied Mathematics \\ Al. I. Cuza 13, Craiova 200585, Dolj, Romania \\ ${ }^{2}$ University of Medicine and Pharmacy of Craiova, IT Department \\ Petru Rareş 2, Craiova 200349, Dolj, Romania
}

\begin{abstract}
The aim of this paper is the study of a dynamical process generated by a sequence of maps:

$x_{n+1}=f_{n}\left(x_{n}\right)$

where $f_{n}:(0, \infty) \rightarrow(0, \infty), f_{n}(x)=\frac{c_{n}}{1+x}$ for all $n \in N$ and $\left(c_{n}\right)_{n}$ is a a sequence of positive numbers.

This process is generated similar to continuous fractions development.

A continued fraction is an expression obtained through an iterative process of representing a number as the sum of its integer part and the inverse of another number, then writing this other number as the sum of its integer part and another inverse, and so on. In a finite continued fraction (or terminated continued fraction), the iteration is terminated after finitely many steps by using an integer in stead of another continued fraction. In contrast, an infinite continued fraction is an infinite expression. In either case, all integers in the sequence, other than the first, must be positive. The integers are called the coefficients or terms of the continued fraction.

We will study the pre-equilibrium points for this process, the attraction basins and the stability.
\end{abstract}

2010 Mathematics Subject Classification: 37B55.

Keywords: Processes; Lyapunov exponent.

\section{Introduction}

We intend to study here a dynamical process derived from the development in continuous fractions of real numbers. The dynamical processes represent the natural generalization of the dynamical systems.

A discrete time dynamical process having the generators $\left(f_{n}\right)_{n}$ is given by the difference equation

$$
x_{n+1}=f_{n}\left(x_{n}\right)
$$

The discrete dynamical systems are particular cases of dynamical processes corresponding to a constant sequence of generators, i.e. $x_{n+1}=f\left(x_{n}\right)$.

\footnotetext{
*e-mail: lilianabucur@yahoo.com

**e-mail: cris_gabriela@yahoo.com
} 
When we model a phenomenon the computer truncates at every step, so we have different functions at each step. We start the study with a certain function, but at each step in reality we will use a new approximation of it. So, it is natural to consider the process defined by $x_{n+1}=f_{n}\left(x_{n}\right)$ instead of a discrete dynamical system $x_{n+1}=f\left(x_{n}\right)$.

In many cases, for example variable step numerical methods, feed-back control schemes it is appropriate to use dynamical processes instead of dynamical systems.

Modeling the behavior of a population exposed to a bacterium or a virus leads to study of a dynamical process because the dynamic of the population changes frequently. At every moment there are people who are healing or others who are getting sick. So, the iteration function is different at each step.

And in economic models rather processes appear.

As in the case of dynamic systems we will study the asymptotic behavior of a process with the help of fixed points. In our study the notions of fixed point, limit circle, basin of attraction, used in the theory of dynamical systems, are adapted for dynamical processes.

\section{Discrete dynamical processes}

Let be (II 1) $x_{n+1}=f_{n}\left(x_{n)}\right.$ for all $n \in \mathbf{N}$ a dynamical process, where $f_{n}: X \rightarrow X$ and $(X, d)$ is a metric space.

Definition 2.1 We call orbit of a point $x_{0} \in X$ the set:

$$
O\left(x_{0}\right)=\left\{x_{0}, f_{0}\left(x_{0}\right), f_{1} \circ f_{0}\left(x_{0}\right), \ldots, f_{n} \circ f_{n-1} \circ \ldots f_{0}\left(x_{0}\right), \ldots\right\}
$$

For a dynamical system is necessary to examine its fixed points to study its asymptotic behavior. So we generalize the fixed point notion for a dynamical process.

Definition 2.2 A point $p \in X$ is called fixed point for the dynamical process (II.1) if and only if $f_{n}(p)=p$ for all $n \in \mathbf{N}$.

This condition seems quite difficult to fulfill.

The approximations made by the computer to every step lead to the idea that, actually, following the discretisation of a continuous system we get rather a process than a dynamical system.

So we will introduce a notion that will prove useful in the study of the asymptotic behavior of a process, that of pre-equilibrium point.

Definition 2.3 A point $p \in X$ is called pre-equilibrium point for the dynamical process (II.1) if and only if

$$
p=\lim _{n \rightarrow \infty} f_{n} \circ f_{n-1} \circ \ldots f_{0}(p)
$$

The attraction basin for a pre-equilibrium point $p$ is the set

$$
B(p)=\left\{x \in X / \lim _{n \rightarrow \infty} f_{n} \circ f_{n-1} \circ \ldots f_{0}(x)=p\right\}
$$

Example 2.1 For a process derived from the logistics application

$$
x_{n+1}=c_{n} x_{n}\left(1-x_{n}\right), \text { for all } n \in N
$$


so $f_{n}(x)=c_{n} x_{n}\left(1-x_{n}\right)$ for all $n \in N$, where $\left(c_{n}\right)_{n}$ decrease to $c>1$, the point $1-\frac{1}{c}$ is a pre-equilibrium point, but is not a fixed point for the process.

Definition 2.4 The system (II.1) is said to be sensitive at a point $x_{0} \in X$ if there exists a constant $\delta>0$ such that for any neighborhood $U \in V\left(x_{0}\right)$, there exists $y_{0} \in U$ and $N \in N$ such that $d\left(f_{N} \circ f_{N-1} \circ \ldots f_{0}\left(y_{0}\right), f_{N} \circ f_{N-1} \circ \ldots f_{0}\left(x_{0}\right)\right)>\delta$.

Definition 2.5 The system (II.1) is said to be stable at a point $x_{0} \in X$ if for any $\varepsilon>0$ there exists a constant $\delta>0$ such that for any $y_{0} \in X$ with $d\left(y_{0}, x_{0}\right)<\delta$ and any $n \in N$ we have $d\left(f_{n} \circ f_{n-1} \circ \ldots f_{0}\left(y_{0}\right), f_{n} \circ f_{n-1} \circ \ldots f_{0}\left(x_{0}\right)\right)<\varepsilon$.

Definition 2.6 If $X$ is a interval and $f_{n}$ are $C^{1}$ maps for any $n \in N$, we define the Lyapunov exponent of the system (II.1) by:

$$
\lambda\left(x_{0}\right)=\limsup _{n \rightarrow \infty} \frac{1}{n} \ln \left|\left(f_{n} \circ f_{n-1} \circ \ldots f_{0}\right)^{\prime}\left(x_{0}\right)\right|=\limsup _{n \rightarrow \infty} \frac{1}{n} \sum_{k=1}^{n} \ln \left|f_{k}^{\prime}\left(x_{k}\right)\right|
$$

where $\left(x_{k}\right)_{k=0, \infty}$ is the orbit of system (II.1) starting from $x_{0}$.

The dynamical process we want to study in this paper is similar to continuous fractions development.

A continuous fraction is an expression of form

$$
a_{0}+\frac{1}{a_{1}+\frac{1}{a_{2}+\frac{1}{a_{3}+}}}
$$

where $a_{0}, a_{1}, \ldots a_{n}, \ldots$ are real numbers.

We will consider $\left(f_{n}\right)_{n}$ where $f_{n}:(0, \infty) \longrightarrow(0, \infty)$,

$$
f_{n}(x)=\frac{c_{n}}{1+x}(I I .2)
$$

and $\left(c_{n}\right)_{n}$ decrease to $c$.

Let be $f(x)=\frac{c}{1+x}$. We have that $\left(f_{n}\right)_{n}$ decrease to $f$.

Let be $x_{n+1}=f_{n}\left(x_{n}\right)$ for all natural $n$.

We will make some simulation:

Let be $c_{n}=4.1+\frac{1}{2^{n}}$

It is obvious that $\left(c_{n}\right)_{n}$ is decreasing to $c=4.1$.

If we consider $x_{0}=0.2$, we obtain

$$
\begin{array}{llll}
x_{0}=0.200000000 & x_{1}=3.83333333 & x_{2}=0.900000000 & x_{3}=2.223684211 \\
x_{4}=1.29122449 & x_{5}=1.803075176 & x_{6}=1.468253522 & x_{7}=1.66425874 \\
x_{8}=1.540355742 & x_{9}=1.614716025 & x_{10}=1.56842130 & x_{11}=1.596501373 \\
x_{12}=1.579141910 & x_{13}=1.58972330 & x_{14}=1.583204270 & x_{15}=1.58718781
\end{array}
$$

We observe that $x_{n}$ is approaching soon to value 1.585665 and stay close. We can consider that the limit $x$ will verify $\left|x_{n}-x\right|<10^{-6}$.

We also notice that $p=\frac{-1+\sqrt{1+4 c}}{2}=1.585665$ in this case.

The graphic is: 


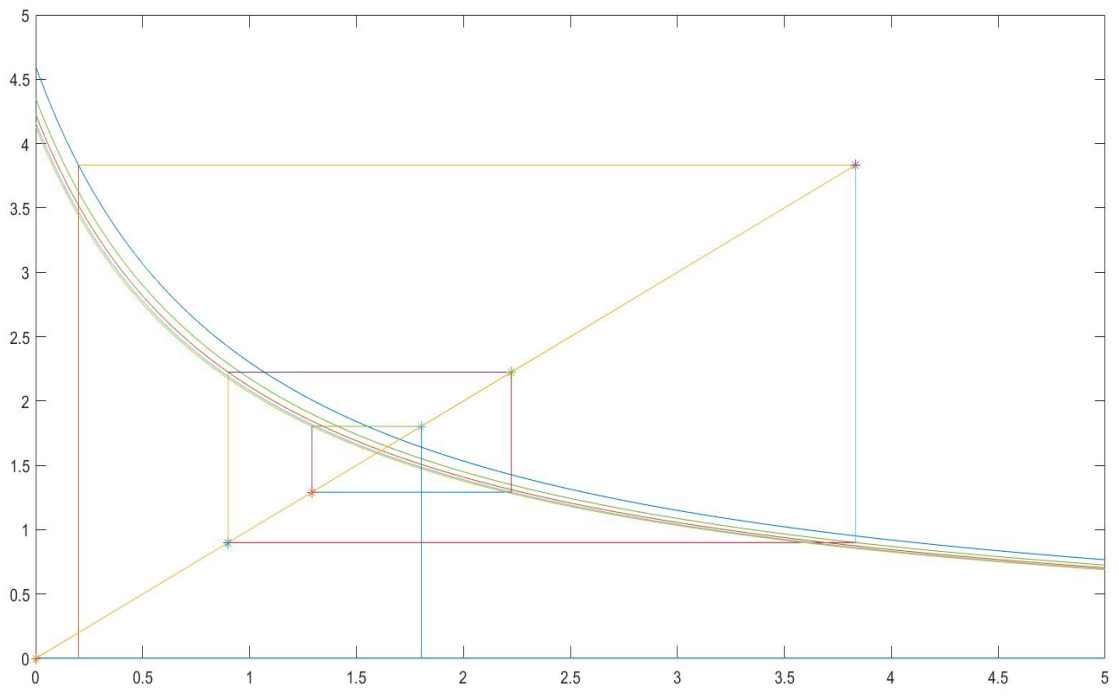

If $x_{0}=0.3$, we obtain the first $15^{\text {th }}$ value:

$\begin{array}{lllll}0.300000000 & 3.538461538 & 0.958474576 & 2.157291216 & 1.318376962 \\ 1.781957839 & 1.479398768 & 1.656777664 & 1.544693147 & 1.611963757 \\ 1.570074068 & 1.595474750 & 1.579766531 & 1.589338423 & 1.583439615 \\ 1.587043295 & 1.584826689 & 1.586182798 & 1.585349581 & 1.585859776 \\ 1.585546514 & 1.585738433 & 1.585620644 & 1.585692831 & 1.585648539 \\ 1.585675690 & 1.585659033 & 1.585669245 & 1.585662981 & 1.585666822 \\ 1.585664466 & 1.585665911 & 1.585665025 & 1.585665235 & 1.585665439 \\ 1.585665314 & 1.585665391 & 1.585665344 & 1.585665372 & 1.585665355 \\ 1.585665366 & 1.585665359 & 1.585665363 & 1.585665361 & 1.585665362 \\ 1.585665361 & 1.585665362 & 1.585665361 & 1.585665362 & 1.585665361\end{array}$

In conclusion, we have also the limit value 1.585665 .

If $x_{0}=0.333$, very close to the previous one we obtain:

$\begin{array}{lllll}0.333333333 & 3.450862716 & 0.977338615 & 2.136710409 & 1.327027190 \\ 1.775333790 & 1.482929735 & 1.654421566 & 1.546064236 & 1.611095693 \\ 1.570596043 & 1.595150780 & 1.579963743 & 1.589216934 & 1.583513912 \\ 1.586997654 & 1.584854649 & 1.586165640 & 1.585360099 & 1.585649098 \\ 1.585675346 & 1.585659244 & 1.585669116 & 1.585663060 & 1.585666773 \\ 1.585664496 & 1.585665892 & 1.585665036 & 1.585665561 & 1.585665239 \\ 1.585665437 & 1.585665315 & 1.585665390 & 1.585665344 & 1.585665372 \\ 1.585665355 & 1.585665365 & 1.585665359 & & \end{array}$

In conclusion, we have also the limit value 1.585665 .

For other values of $x_{0}$ we have: $x_{0}=0.578086881$

$\begin{array}{lllll}2.914921894 & 1.111133279 & 2.001294775 & 1.386901425 & 1.730800425 \\ 1.507113066 & 1.638463201 & 1.555415383 & 1.605200138 & 1.574150294 \\ 1.592948279 & 1.581305795 & 1.588390681 & 1.584019393 & 1.586687209 \\ 1.585044858 & 1.586048930 & 1.585652905 & 1.585673012 & 1.585660675 \\ 1.585668238 & 1.585663599 & 1.585666443 & 1.585664698 & 1.585665768 \\ 1.585665112 & 1.585665514 & 1.585665268 & 1.585665419 & 1.585665326\end{array}$




\begin{tabular}{ccccc}
1.585665383 & 1.585665348 & 1.585665370 & 1.585665356 & 1.585665365 \\
1.58566536 & & & & \\
$x_{0}=0.91$ & & & & \\
2.408376963 & 1.276267281 & 1.856108918 & 1.457402403 & 1.681145097 \\
1.535025093 & 1.620422816 & 1.566123690 & 1.598501717 & 1.57820814 \\
1.590441135 & 1.582836253 & 1.587449481 & 1.584595589 & 1.586333481 \\
1.585261641 & 1.585915934 & 1.585657242 & 1.585670352 & 1.585662307 \\
1.585667238 & 1.585664212 & 1.585666067 & 1.585664929 & 1.585665627 \\
1.585665199 & 1.585665461 & 1.585665300 & 1.585665399 & 1.585665338 \\
1.585665376 & 1.585665353 & 1.585665367 & 1.585665358 & 1.585665363 \\
1.58566536 & & & & \\
$x_{0}=0.92$ & & & & \\
2.395833333 & 1.280981595 & 1.852272727 & 1.459362550 & 1.679805200 \\
1.535792602 & 1.619932363 & 1.566416870 & 1.598319109 & 1.578319056 \\
1.590372717 & 1.582878060 & 1.587423787 & 1.584611325 & 1.586323823 \\
1.585267561 & 1.585912302 & 1.585515414 & 1.585670280 & 1.585662351 \\
1.585667210 & 1.585664229 & 1.585666057 & 1.585664935 & 1.585665623 \\
1.585665201 & 1.585665460 & 1.585665301 & 1.585665398 & 1.585665339 \\
1.585665375 & 1.585665353 & 1.585665367 & 1.585665358 & 1.585665363 \\
1.585665360 & 1.585665362 & & & \\
\hline
\end{tabular}

In conclusion, more values of $x_{0}$ lead to the same limit $1.585665=p=\frac{-1+\sqrt{1+4 c}}{2}$.

Let be $c_{n}=5.1+\frac{1}{2^{n}}$

It is obvious that $\left(c_{n}\right)_{n}$ is decreasing to $c=5.1$.

If we consider $x_{0}=0.73$, we obtain

$x_{0}=0.732210118$

$\begin{array}{lllll}3.232864155 & 1.263919607 & 2.307944144 & 1.560636992 & 2.003895912 \\ 1.702996758 & 1.889685026 & 1.766250025 & 1.844357191 & 1.793367085 \\ 1.825928396 & 1.804803033 & 1.818353022 & 1.809589145 & 1.815222886 \\ 1.811584896 & 1.813926244 & 1.812415597 & 1.813388431 & 1.812761046 \\ 1.813165212 & 1.812904630 & 1.813072532 & 1.812964295 & 1.813034043 \\ 1.812989085 & 1.813018058 & 1.812999383 & 1.813011419 & 1.813003661 \\ 1.813008661 & 1.813005438 & 1.813007515 & 1.813006177 & 1.813007039 \\ 1.813006483 & 1.813006842 & 1.813006611 & 1.813006760 & 1.813006664 \\ 1.813006725 & 1.813006686 & 1.813006711 & 1.813006695 & 1.813006705 \\ 1.813006699 & 1.813006703 & 1.813006700 & 1.813006702 & \end{array}$

$x_{0}=0.33$

4.210526316

1.649924433

1.026767677

2.577996511

1.442846572

2.100520786

1.832242773

1.927531380

1.743416410

1.859707883

1.783740428

1.800779294

1.820965358

1.807913387

1.816306209

1.810888050

1.814375933

1.812126004

1.813575174

1.812640729

1.813242774

1.812854648

1.813104749

1.812943532

1.813047426

1.812980460

1.813023617

1.812995800

1.813013728

1.813002173

1.813009620

1.813004820

1.813007914

1.813005920

1.813007205

1.813006377

1.813006910

1.813006566

1.813006788

1.813006645

1.813006737

1.813006678

1.813006716

1.813006692

1.813006707

\subsection{7}

1.813006704

1.813006700

1.813006702

So, we obtain the limit 1.813006 ho is equal with $p=\frac{-1+\sqrt{1+4 c}}{2}$.

We can resume in the next theoretical result: 
Proposition 2.1 The process (II.2) has no fixed points, but has a pre-equilibrium point:

$$
p=\frac{-1+\sqrt{1+4 c}}{2}
$$

Moreover, the attraction basin of this point is the interval $(0, \infty)$.

Proof. Let be

$$
p_{n}=\frac{-1+\sqrt{1+4 c_{n}}}{2}
$$

It is obvious that $\left(p_{n}\right)_{n}$ is decreasing to $p$.

Let be $y_{1}=p$ and $y_{n+1}=f_{n}\left(y_{n}\right)$ for all $n \geq 1$.

We will prove that the sequence $\left(y_{n}\right)_{n}$ converge to $p$.

Let observe that for all natural $n$ :

- if $x \leq p_{n}$ we have $f_{n}(x) \geq x$ and $f_{n}(x) \geq p_{n}$,

- if $x \geq p_{n}$ then $f_{n}(x) \leq x$ and $f_{n}(x) \leq p_{n}$.

Case 1. If $y_{n} \geq p_{n}$ for all $n \geq N$. Then we have $f_{n}\left(y_{n}\right) \leq y_{n}$, so $y_{n+1} \leq y_{n}$ for all $n \geq N$. So, $\left(y_{n}\right)_{n}$ is decreasing and positive that means $\left(y_{n}\right)_{n}$ will converge to

a limit $y$.

Because $y_{n+1}=f_{n}\left(y_{n}\right)$ due to the Dini's lemma we get $f(y)=y$, so $y=p$, the only fixed point of $f$.

Case 2. There exists a smallest $N \in N$ such that $y_{N}<p_{N}$ then $f_{N}\left(y_{N}\right) \geq p_{N}$. That means $y_{n} \geq p_{n}$ for all $n \geq N$, so we have Case 1 again.

Case 3 . There is a sous- sequence $\left(y_{k_{n}}\right)_{k_{n}}$ such that $y_{k_{n}} \leq p_{k_{n}}$ for all $n \in N$.

We will use the next observation:

(II.3) If $x<\sqrt{1+4 c}$, then $x<\frac{c_{n}(\sqrt{1+4 c}+1)}{c}$ for all $n \in N$, that means $f_{n}(x) \geq p$.

Due of this observation we have $p<y_{k_{n}} \leq p_{k_{n}}$, so $\left(y_{k_{n}}\right)_{k_{n}}$ will converge to $p$.

In conclusion, sequence $\left(y_{n}\right)_{n}$ converge to $p$, so

$$
p=\lim _{n \rightarrow \infty} f_{n} \circ f_{n-1} \circ \ldots f_{0}(p)
$$

and $p$ will be pre-equilibrium point.

In a similary way we can prove that if $y_{1} \leq \sqrt{1+4 c}$ then $\lim _{n \rightarrow \infty} f_{n} \circ f_{n-1} \circ \ldots f_{0}\left(y_{1}\right)=p$, so $y_{1} \in B(p)$.

If $y_{1} \geq \sqrt{1+4 c}$ then $f_{1}\left(y_{1}\right) \leq \sqrt{1+4 c}$, that means $y_{2} \leq \sqrt{1+4 c}$ then $\lim _{n \rightarrow \infty} f_{n} \circ f_{n-1} \circ$ $\ldots f_{0}\left(y_{1}\right)=p$.

In conclusion, $B(p)=(0, \infty)$.

If we consider $c_{n}=\left(1+\frac{1}{n}\right)^{n+1}$ we have

$\begin{array}{lllll}1.000000000 & 2.000000000 & 1.125000000 & 1.487291213 & 1.226940294 \\ 1.340846006 & 1.256766753 & 1.289581816 & 1.260713881 & 1.268613430 \\ 1.257647807 & 1.258364731 & 1.253468136 & 1.252374296 & 1.249705808 \\ 1.248342764 & 1.246606644 & 1.245367328 & 1.244094649 & 1.243044637 \\ 1.242045096 & 1.241166801 & 1.240350394 & 1.239612048 & 1.238928886 \\ 1.238301851 & 1.237720497 & 1.237182138 & 1.236680996 & 1.236213998 \\ 1.235777407 & 1.235368549 & 1.234984751 & 1.234623841 & 1.234283797 \\ 1.233962879 & 1.233659507 & 1.233372287 & 1.233099959 & 1.232841396 \\ 1.232595580 & 1.232361590 & 1.232138595 & 1.231925836 & 1.231722624 \\ 1.231528332 & 1.231342385 & 1.231164256 & 1.230993462 & 1.230829560 \\ 1.230672141 & 1.230520827 & 1.230375270 & 1.230235148 & 1.230100163\end{array}$




$\begin{array}{lllll}1.229970036 & 1.229844509 & 1.229723345 & 1.229606318 & 1.229493220 \\ 1.229383857 & 1.229278048 & 1.229175620 & 1.229076416 & 1.228980284 \\ 1.228887086 & 1.228796687 & 1.228708964 & 1.228623801 & 1.228541086 \\ 1.228460715 & 1.228382591 & 1.228306620 & 1.228232714 & 1.228160791 \\ 1.228090772 & 1.228022581 & 1.227956149 & 1.227891408 & 1.227828295 \\ 1.227766749 & 1.227706711 & 1.227648129 & 1.227590948 & 1.227535120 \\ 1.227480596 & 1.227427333 & 1.227375286 & 1.227324414 & 1.227274679\end{array}$

The limit will be $1.227=p$, so $\mathrm{c}$ will be 2,7182 , so we find the approximation of the number $e$.

Let be $y_{0}=p=\frac{-1+\sqrt{1+4 c}}{2}$, the pre-equilibrium point.

$$
\lambda\left(y_{0}\right)=\limsup _{n \rightarrow \infty} \frac{1}{n} \ln \left|\left(f_{n} \circ f_{n-1} \circ \ldots f_{0}\right)^{\prime}\left(y_{0}\right)\right|=\limsup _{n \rightarrow \infty} \frac{1}{n} \sum_{k=1}^{n} \ln \left|f_{k}^{\prime}\left(y_{k}\right)\right|
$$

where $\left(y_{k}\right)_{k=0, \infty}$ is the orbit of system (II.2) becoming from $y_{0}$.

We already proved that $\left(y_{k}\right)_{k}$ converge to $p$, so

$$
\begin{gathered}
\limsup _{n \rightarrow \infty} \frac{1}{n} \ln \left|\left(f_{n} \circ f_{n-1} \circ \ldots f_{0}\right)^{\prime}\left(y_{0}\right)\right|=\limsup _{n \rightarrow \infty} \frac{1}{n} \sum_{k=1}^{n} \ln \left|f_{k}^{\prime}\left(y_{k}\right)\right|=\limsup _{n \rightarrow \infty} \frac{1}{n} \ln \prod_{k=1}^{n}\left|f_{k}^{\prime}\left(y_{k}\right)\right| \\
\lambda\left(y_{0}\right)=\ln \limsup _{n \rightarrow \infty} \sqrt[n]{\prod_{k=1}^{n}\left|f_{k}^{\prime}\left(y_{k}\right)\right|}=\ln \limsup _{n \rightarrow \infty}\left|f_{n+1}^{\prime}\left(y_{n+1}\right)\right|=\ln \frac{c}{(1+p)^{2}}=\ln \frac{1+2 c+\sqrt{1+4 c}}{2 c}
\end{gathered}
$$

Using the Theorem 3.1 from [2] because $\lambda\left(y_{0}\right)>0$ the process will be sensitive in $y_{0}$.

\section{References}

[1] M.L. Bucur, Dynamical processes. Stability and chaos, Differential Geometry - Dynamical Systems, Balkan Society of Geometers, Geometry Balkan Press 21, 47-51 (2019).

[2] M.L. Bucur, Procese dinamice. Puncte de pre-echilibru, Volumul Simpozionului International Universul Ştiintelor, Ediţia a IV-a, 423-426 (2013).

[3] H. Shao, Y. Shi, H. Zhu, Lyapunv exponents, sensitivity and stability for non-autonomous systems, arXiv: math. DS/1603.05457v 17 mar 2016.

[4] T. Nesemann, Invariants and Liapunov Functions for Nonautonomous Systems, Computers and Mathematics with Applications 42 (2001).

[5] E. Kaslik, and St. Balint, On the controllability of some steady states in the case of nonlinear discrete dynamical systems with control, arXiv:math. DS/031116v1 11 Nov 2003.

[6] A. Radwan, On some generalized discrete logistic maps, Journal of Advanced Research 4, 163-171 (2013). 